\title{
ON EQUIVALENCE OF GRADED RINGS
}

\author{
MASHHOOR REFAI* \\ Department of Mathematics and Computer Science \\ Faculty of Science, P O. Box 17551 \\ United Arab Emirates University \\ Al-Ain, UNITED ARAB EMIRATES
}

\section{SOFYAN OBIEDAT}

\author{
Department of Mathematics \\ Yarmouk University \\ Irbid, JORDAN
}

(Received April 14, 1995 and in revised form May 2, 1996)

\begin{abstract}
Let $R=\underset{g \in G}{\oplus} R_{g}$ be a $G$-graded ring. In this paper we define the "homogeneousequivalence" concept between graded rings We discuss some properties of the $G$-graded rings and investigate which of these are preserved under homogeneous-equivalence maps. Furthermore, we give some results in graded ring theory and also some applications of this concept to $Z$-graded rings
\end{abstract}

KEY WORDS AND PHRASES: Graded rings, homogeneously-equivalent graduations, $Z$-graded rings.

1991 AMS SUBJECT CLASSIFICATION CODES: 16A03

\section{INTRODUCTION}

Let $G$ be a group with identity $e$. Then a ring $R$ is said to be $G$-graded ring if there exist additive subgroups $R_{g}$ of $R$ such that $R=\underset{g \in G}{\oplus} R_{g}$ and $R_{g} R_{h} \subseteq R_{g h}$ for all $g, h \in G$. We consider $\operatorname{supp}(R)=\left\{g \in G: R_{g} \neq 0\right\}$. The elements of $R_{g}$ are called homogeneous of degree $g$. If $x \in R$, then $x$ can be written uniquely as $\sum_{g \in G} x_{g}$ where $x_{g}$ is the component of $x$ in $R_{g}$. Also, we write $h(R)=\bigcup_{\mathbf{g} \in \mathrm{G}} R_{g}$

In this paper we define an equivalence relation on the set of all graded rings and give some applications of this relation. In Section 1, we define the homogeneous-equivalence concept between graded rings and give the necessary and sufficient conditions for two graded rings to be homogeneouslyequivalent. In Section 2, we discuss the relation between this new concept and some related concepts given in [1] In Section 3, we give some properties of graded rings and see which of these are preserved under homogeneous-equivalence maps. In Section 4, we give some useful results and applications of this new concept to $Z$-graded rings. We give the necessary and sufficient conditions for two first strongly $Z$-graded rings to be homogeneously-equivalent.

\section{HOMOGENEOUS-EQUIVALENCE OF GRADUATIONS}

In this section we define the homogeneous-equivalence concept between graded rings and give some of its properties. Also, we give the necessary and sufficient conditions for two graded rings to be homogeneously-equivalent.

DEFINITION 1.1. Let $G, H$ be groups, $R$ be a $G$-graded ring and $S$ be an $H$-graded ring. We say that $R$ is homogeneously equivalent (shortly "h.e.") to $S$ if there exists a ring isomorphism $f: R \rightarrow S$ sending $h(R)$ onto $h(S)$. We call such an $f$ a homogeneous-equivalence of $R$ with $S$

From now on $G, H$ are groups, $R$ is a G-graded ring and $S$ is an $H$-graded ring unless otherwise indicated. 
REMARK 1.2. The relation h.e. is an equivalence relation.

PROPOSITION 1.3. An isomorphism $f: R \rightarrow S$ of rings is a homogeneous equivalence if and only if there is a bijection $b$ of supp $(R)$ onto supp $(S)$ such that $f\left(R_{g}\right)=S_{b(g)}$ for all $g \in \operatorname{supp}(R)$ In that case the bijection $b$ is uniquely determined by the isomorphism $f$ If $R \neq 0$, then the bijection $b$ sends the identity element $e_{G} \in \operatorname{supp}(R)$ of $G$ onto the identity element $e_{H} \in \operatorname{supp}(S)$ of $H$.

PROOF. Suppose $f: R \rightarrow S$ is a homogeneous equivalence map. Define $b: \operatorname{supp}(R) \rightarrow \operatorname{supp}(S)$ by $b(g)=h$ where $f\left(R_{g}\right)=S_{h}$. Then clearly $b$ is a well-defined bijective map. The converse is obvious

Suppose $R \neq 0$. To show that $b\left(e_{G}\right)=e_{H}$ it is enough to show $f\left(R_{e G}\right)=S_{e_{H}}$. Since $e_{H} \in H$, there exists $g \in G$ such that $f\left(R_{g}\right)=S_{e_{H}}$ and then $f\left(R_{g}\right) f\left(R_{g}\right)=S_{e_{H}}$, i.e., $S_{e_{H}} \subseteq f\left(R_{g^{2}}\right)$. Therefore, $f\left(R_{g^{2}}\right)=S_{e_{H}}$. But $f$ is 1-1 implies $R_{g}=R_{g^{2}} \neq 0$. Hence $g=g^{2}$ and then $g=e_{G}$, i.e., $f\left(R_{e_{G}}\right)=S_{e_{H}}$

Let $I$ be an ideal of a graded ring $R$. Then $I$ is a graded ideal of $R$ if $I=\underset{g \in G}{\oplus}\left(R_{g} \cap I\right)$.

PROPOSITION 1.4. Suppose $R$ is h.e. to $S$ by $f$. Then $I$ is a graded ideal of $R$ if and only if $f(I)$ is a graded ideal of $S$.

PROOF. An ideal $I$ in a $G$-graded ring $R$ is graded if and only if it is generated as an ideal by its subset $I \cap h(R)$. Obviously this property is preserved under homogeneous equivalence maps.

REMARK 1.5. It follows from Proposition 1.4 that any property definable solely in terms of graded ideals of $R$ must be preserved under homogeneous equivalence maps.

PROPOSITION 1.6. Let $R$ be h.e. to $S$ by $f$ and $I$ be a graded ideal of $R$. Then $R / I$ is h.e. to $S / f(I)$.

PROOF. By Proposition 1.4, $f(I)$ is a graded ideal of $S$ and then $S / f(I)$ is an $H$-graded ring Define $\varphi: R / I \rightarrow S / f(I)$ by $\varphi(r+I)=f(r)+f(I)$. Then clearly $\varphi$ is a ring isomorphism. Let $h \in \operatorname{supp}(S / f(I))$. Then $(S / f(I))_{h} \neq 0$ and hence there exists $s_{h} \in S_{h}-f(I)$, i.e., $h \in \operatorname{supp}(S)$ Since $R$ is h.e. to $S$ by $f$, there exists $g \in \operatorname{supp}(R)$ such that $f\left(R_{g}\right)=S_{h}$ by Proposition 1.3

CLAIM. $\varphi\left((R / I)_{g}\right)=(S / f(I))_{h}$.

Let $r_{g}+I \in(R / I)_{g}$. Then $\varphi\left(r_{g}+I\right)=f\left(r_{g}\right)+f(I) \in(S / f(I))_{h}$. So, $\varphi\left((R / I)_{g}\right) \subseteq(S / f(I))_{h}$. Conversely, let $t_{h}+f(I) \in(S / f(I))_{h}$. Then $f^{-1}\left(t_{h}\right) \in R_{g}$ and hence $f^{-1}\left(t_{h}\right)+I \in(R / I)_{g}$. So, $\varphi\left(f^{-1}\left(t_{h}\right)+I\right)=t_{h}+f(I) \in \varphi\left((R / I)_{g}\right)$. Therefore, $\varphi\left((R / I)_{g}\right)=(S / f(I))_{h}$ and hence $R / I$ is h.e to $S / f(I)$

\section{VARIOUS EQUIVALENCE OF GRADUATIONS}

In this section we discuss the relation between the homogeneous-equivalence concept and the equivalence and almost-equivalence concepts given in [1]

DEFINITION 2.1 ([1]). We say that $R$ is almost equivalent (shortly "a.e.") to $S$ if there exists a ring isomorphism $f: R \rightarrow S$ such that for each $h \in H$, there exists $g \in G$ with $f\left(R_{g}\right)=S_{h}$. If $R$ is a.e. to $S$ and $S$ is a.e. to $R$ then we say $R$ is equivalent to $S$.

PROPOSITION 2.2. If $R$ is a.e. to $S$ then $R$ is h.e. to $S$.

PROOF. Follows from Proposition 1.3

However, the converse of this proposition need not be true in general as we see in the following example.

EXAMPLE 2.3. Let $K$ be a field and $R=S=K[x]$ is the polynomial ring over $K$ in one variable $x$. Let $G=Z_{3}$. Then $R$ is a $G$-graded ring with $R_{\jmath}=\left\{k x^{3 r+\jmath}: k \in K, r=0,1, \ldots\right\}$ for $j \in Z_{3}$. Let $H=Z_{6}$. Then $S$ is an $H$-graded ring with

$$
\begin{aligned}
& S_{0}=\left\{k x^{3 r}: k \in K, r=0,1, \ldots\right\} \\
& S_{2}=\left\{k x^{3 r+1}: k \in K, r=0,1, \ldots\right\} \\
& S_{4}=\left\{k x^{3 r+2}: k \in K, r=0,1, \ldots\right\} \text { and } S_{\jmath}=0 \text { otherwise. }
\end{aligned}
$$


Clearly $R$ is h.e. to $S$. If $R$ is a.e. to $S$ by $f$ then there exists $g \in G$ with $f\left(R_{g}\right)=S_{3}=0$, i e, $R_{g}=0$ a contradiction.

PROPOSITION 2.4. $R$ is a.e to $S$ if and only if the following two conditions are satisfied:

(i) $R$ is h.e. to $S$

(ii) If $H$-supp $(S) \neq \emptyset$ then $G$-supp $(R) \neq \emptyset$

PROOF. Suppose $R$ is a.e. to $S$ by $f$. Then (i) follows from Proposition 2.2 Assume $H$-supp $(S) \neq \emptyset$. Then there exists $h \in H$ such that $S_{h}=0$. Since $R$ is a.e to $S$ there exists $g \in G$ such that $f\left(R_{g}\right)=0$. Thus $R_{g}=0$, i.e., $G$-supp $(R) \neq \emptyset$.

Conversely, suppose $R$ is h.e. to $S$ by $f$. Let $h \in H$. If $h \in \operatorname{supp}(S)$ then by Proposition 1.3, there exists $g \in G$ such that $f\left(R_{g}\right)=S_{h}$. If $h \notin \operatorname{supp}(S)$ then by (ii) there exists $g \notin \operatorname{supp}(R)$ So, $0=f\left(R_{g}\right)=S_{h}$, i.e., $R$ is a.e to $S$ by $f$.

COROLLARY 2.5. $R$ is equivalent to $S$ if and only if the following two conditions are satisfied.

(i) $R$ is h.e. to $S$.

(ii) $G$-supp $(R) \neq \emptyset$ if and only if $H$-supp $(S) \neq \emptyset$.

PROPOSITION 2.6. Suppose $R$ is h.e. to $S$ and $|G|=|H|=n<\infty$. Then $R$ is equivalent to $S$.

PROOF. Similar to the proof of Proposition 2.3 in [1].

\section{PROPERTIES PRESERVED UNDER HOMOGENEOUS-EQUIVALENCE MAPS}

In this section we give some properties of graded rings and see which of these are preserved under homogeneous-equivalence maps. For more details about the properties one can look in $[1,2,3]$.

DEFINITION 3.1. For a $G$-graded ring $R$ we say

1. $R$ is semiprime if $R$ has no non-zero nilpotent graded ideals.

2. $R$ is prime if the product of any two non-zero graded ideals (of the same type right or left) of $R$ is non-zero.

3. $R$ is Noetherian (Artinian) if $R$ satisfies the ascending (descending) chain conditions on graded ideals of $R$.

4. $R$ is simple if $O, R$ are the only graded ideals of $R$.

PROPOSITION 3.2. Suppose $R$ is h.e. to $S$. Then

1. $R$ is semiprime if and only if $S$ is semiprime.

2. $R$ is prime if and only if $S$ is prime

3. $R$ is Noetherian (Artinian) if and only if $S$ is Noetherian (Artinian).

4. $R$ is simple if and only if $S$ is simple.

PROOF. Follows from Remark 1.5.

DEFINITION 3.3. For a $G$-graded ring $R$ we say

1. $R$ is strong if $R_{g} R_{h}=R_{g h}$ for all $g, h \in G$. Also, $R$ is strong if $1 \in R_{g} R_{g-1}$ for all $g \in G$ (Proposition 1.6 of [4]).

2. $R$ is first strong if $1 \in R_{g} R_{g-1}$ for all $g \in \operatorname{supp}(R)$.

$3 R$ is second strong if $R_{g} R_{h}=R_{g h}$ for all $g, h \in \operatorname{supp}(R)$ and $\operatorname{supp}(R)$ is a monoid in $G$.

It is easy to see that a $G$-graded ring $R$ is first strong if and only if $\operatorname{supp}(R)$ is a subgroup of $G$ and $R$ is strong as a $\operatorname{supp}(R)$-graded ring. It follows that the bijection $b: \operatorname{supp}(R) \rightarrow \operatorname{supp}(S)$ given in Proposition 1.3 which is induced by a homogeneous-equivalence $f$ of $R$ onto an $H$-graded ring $S$ is an isomorphism of groups whenever $R$ is first strong. Similarly any such $f$ induces an isomorphism $b$ of $\operatorname{supp}(R)$ onto $\operatorname{supp}(S)$ as monoids whenever $R$ is second strong. So, we have the following results.

PROPOSITION 3.4. Suppose $R$ is h.e. to $S$. Then

1. $R$ is first strong if and only if $S$ is first strong.

2. $R$ is second strong if and only if $S$ is second strong.

3. If $R$ is strong then $\operatorname{supp}(S)$ is a subgroup of $H$ and $G$ is isomorphic to $\operatorname{supp}(S)$ as groups. 
DEFINITION 3.5. A $G$-graded ring $R$ is nondegenerate if for any $a_{g} \in R_{g}-0, a_{g} R_{g^{-1}} \neq 0$ and $R_{g^{-1}} a_{g} \neq 0$.

PROPOSITION 3.6. Suppose $R$ is h.e. to $S$ Then $R$ is nondegenerate if and only if $S$ is nondegenerate.

PROOF. Similar to the proof of Proposition 2.7 in [1].

DEFINITION 3.7. A $G$-graded ring $R$ is regular if $a_{g} \in a_{g} R_{g^{-1}} a_{g}$ for all $g \in G$ and $a_{g} \in R_{g}$

PROPOSITION 3.8. Suppose $R$ is h.e. to $S$. Then $R$ is regular if and only if $S$ is regular.

PROOF. Suppose $R$ is h.e. to $S$ by $f$, and $R$ is regular. Let $a_{h} \in S_{h}$ If $a_{h}=0$ then $a_{h} \in a_{h} S_{h^{-1}} a_{h}$. If $a_{h} \neq 0$, then there exists $g \in \operatorname{supp}(R)$ and a non-zero element $b_{g} \in R_{g}$ such that $f\left(b_{g}\right)=a_{h}$. Thus, $a_{h}=f\left(b_{g}\right) \in f\left(b_{g} R_{g^{-1}} b_{g}\right)=a_{h} S_{h^{-1}} a_{h}$, i.e., $S$ is regular. The other part is obvious since h.e. is symmetric.

DEFINITION 3.9. A $G$-graded ring $R$ is faithful if for any $a_{g} \in R_{g}-0, a_{g} R_{h} \neq 0$ and $R_{h} a_{g} \neq 0$ for all $g, h \in G$.

In the following example we show that faithfulness and strongness need not be preserved under homogeneous-equivalence maps. .

EXAMPLE 3.10. Let $R=S=Z[i]=\{a+i b: a, b \in Z\}$ (The Gaussian integers). Let $G=Z_{2}$ and $H=Z_{4}$. Then $R$ is a $G$-graded ring with $R_{0}=Z, R_{1}=i Z$. Also, $S$ is an $H$-graded ring with $S_{0}=Z, S_{2}=i Z$ and $S_{1}=S_{3}=0$.

Clearly $R$ is h.e. to $S$. But $R$ is faithful and strong while $S$ is not strong because $S_{1} S_{1} \neq S_{2}$. Moreover, $S$ is not faithful because $i \in S_{2}-0$ and $i S_{3}=0$.

\section{APPLICATIONS TO Z-GRADED RINGS}

In this section we give necessary and sufficient conditions for two first strongly $Z$-graded rings to be homogeneously-equivalent. Also, we define homogeneous-equivalence order-preserving maps between $Z$-graded rings.

PROPOSITION 4.1. Let $R$ and $S$ be first strong graduations and assume that $\operatorname{supp}(R), \operatorname{supp}(S)$ are cyclic groups. Then $R$ is h.e. to $S$ if and only if there exists a ring isomorphism $f: R \rightarrow S$ and there exists generator $g$ of $\operatorname{supp}(R)$ and generator $h$ of $\operatorname{supp}(S)$ such that $f\left(R_{g}\right)=S_{h}$ and $f\left(R_{g^{-1}}\right)=S_{h^{-1}}$.

PROOF. The first part is obvious because homogeneous-equivalence of first strong graded rings induce isomorphisms between their support subgroups. Suppose $f: R \rightarrow S$ is a ring isomorphism. Let $g, h$ be generators of $\operatorname{supp}(R), \operatorname{supp}(S)$ respectively with $f\left(R_{g}\right)=S_{h}$ and $f\left(R_{g^{-1}}\right)=S_{h^{-1}} \quad$ Let $h^{\prime} \in \operatorname{supp}(S)$. Then there exists $n \in Z$ such that $h^{\prime}=h^{n}$. If $n=0$, then $h^{\prime}=e_{H}$ and hence $f\left(R_{e G}\right)=f\left(R_{g} R_{g^{-1}}\right)=S_{h} S_{h^{-1}}=S_{e_{H}}=S_{h^{\prime}}$. If $n>0$ then $S_{h^{\prime}}=S_{h} S_{h} \ldots S_{h}$ ( $n$-times), and hence $S_{h^{\prime}}=f\left(R_{g^{n}}\right)$. If $n<0$ then $S_{h^{\prime}}=S_{h^{-1}} S_{h^{-1}} \ldots S_{h^{-1}}((-n)$-times $)$, and hence $S_{h^{\prime}}=f\left(R_{g^{n}}\right)$. Therefore, $R$ is h.e. to $S$ by $f$.

DEFINITION 4.2. Let $R$ be a $Z$-graded ring. Then $R$ is said to be

(1) Right limited if there exists $j \in Z$ such that $R_{h}=0$ for all $i \geq j$.

(2) Left limited if there exists $j \in Z$ such that $R_{\imath}=0$ for all $i \leq j$

The property of right and left limited are not preserved between h.e. $Z$-graded rings as we see in the following example.

EXAMPLE 4.3. Let $K$ be a field and $R=K[x]$ is the polynomial ring over $K$ in one variable $x$ Then $R$ is a $Z$-graded ring with $R_{0}=K, R_{i}=K x^{2}$ for $i>0$ and $R_{h}=0$ for $i<0$.

Let $S=K[x]$. Then $S$ is a $Z$-graded ring with $S_{0}=K, S_{\jmath}=K x^{-j}$ for $j<0$ and $S_{\jmath}=0$ for $j>0$. Clearly $R$ is h.e. to $S$; $R$ is left limited while $S$ is not left limited.

Now, we will add extra conditions to ensure that these properties are preserved between h.e $Z$ graded rings. 
DEFINITION 4.4. Let $R$ and $S$ be $Z$-graded rings such that $R$ is he. to $S$ by $f$. Then $f$ is orderpreserving map if whenever $r, s \in h(R)-0$ with $\operatorname{deg}(r)<\operatorname{deg}(s)$ we have $\operatorname{deg}(f(r))<\operatorname{deg}(f(s))$

\section{REMARKS 4.5.}

1) $f$ is an order-preserving map if and only if $f^{-1}$ is order-preserving

2) The map $f$ which shows $R$ is h.e. to $S$ in Example 4.3, is not an order-preserving map.

DEFINITION 4.6 ([5]) Let $R$ be a $Z$-graded ring and $I$ be an ideal of $R$

1) We denote by $I^{\sim}$ the ideal of $R$ generated by $\left\{r \in h(R):\right.$ there exists $x \in I$ with $x=x_{1}+\ldots+x_{n}+r$;

$x_{\imath} \in R_{s_{1}}-0, s_{\imath} \in Z$ and $\left.\operatorname{deg}\left(x_{1}\right)<\operatorname{deg}\left(x_{2}\right)<\ldots<\operatorname{deg}\left(x_{n}\right)<\operatorname{deg}(r)\right\}$.

2) We denote by $I_{\sim}$ the ideal of $R$ generated by $\left\{r \in h(R):\right.$ there exists $x \in I$ with $x=r+x_{1}+\ldots+x_{n}$;

$$
\left.x_{\imath} \in R_{s_{1}}-0, s_{\imath} \in Z \text { and } \operatorname{deg}(r)<\operatorname{deg}\left(x_{1}\right)<\operatorname{deg}\left(x_{2}\right)<\ldots<\operatorname{deg}\left(x_{n}\right)\right\} .
$$

One can show that $I^{\sim}$ and $I_{\sim}$ are graded ideals. Moreover, if $I, J$ are ideals of $R$ with $I \subseteq J$ then $I^{\sim} \subseteq J^{\sim}$.

PROPOSITION 4.7. Suppose $R$ and $S$ are $Z$-graded rings such that $R$ is h.e. to $S$ by an orderpreserving map $f$. Let $I$ be an ideal of $R$. Then

1) $f\left(I^{\sim}\right)=(f(I))^{\sim}$

2) $f\left(I_{\sim}\right)=(f(I))_{\sim}$

3) $(R / I)^{\sim}$ is h.e. to $(S / f(I))^{\sim}$ and

$(R / I)_{\sim}$ is h.e. to $(S / f(I))_{\sim}$.

PROOF. 1) Let $I^{\sim}$ be the ideal of $R$ generated by $A=\{a \in h(R)$ : there exists $x \in I$ with $x=x_{1}+x_{2}+\ldots+x_{n}+a ; x_{\imath} \in R_{s_{1}}-0, s_{\imath} \in Z$ and $\left.\operatorname{deg}\left(x_{1}\right)<\operatorname{deg}\left(x_{2}\right)<\ldots<\operatorname{deg}\left(x_{n}\right)<\operatorname{deg}(a)\right\}$. Let $a \in A$ and assume $x=x_{1}+x_{2}+\ldots+x_{n}+a$. Then $f(x)=f\left(x_{1}\right)+f\left(x_{2}\right)+\ldots+f\left(x_{n}\right)+f(a)$. Since $f$ is an order-preserving map, $\operatorname{deg}\left(f\left(x_{1}\right)\right)<\operatorname{deg}\left(f\left(x_{2}\right)\right)<\ldots<\operatorname{deg}\left(f\left(x_{n}\right)\right)<\operatorname{deg}(f(a))$. But $f(x) \in f(I)$ implies $f(a) \in(f(I))^{\sim}$. Therefore, $f(A) \subseteq(f(I))^{\sim}$ and hence $f\left(I^{\sim}\right) \subseteq(f(I))^{\sim}$ Also, $(f(I))^{\sim} \subseteq f\left(I^{\sim}\right)$ since $f^{-1}$ is an order-preserving map.

2) Similar to 1).

3) Follows from 1), 2) and Proposition 1.6.

PROPOSITION 4.8. Suppose $R$ and $S$ are $Z$-graded rings such that $R$ is h.e. to $S$ by an orderpreserving map $f$. Then $R$ is right (left) limited if and only if $S$ is right (left) limited.

PROOF. Suppose $R$ is right limited. Then there exists $k \in Z$ such that $R_{\imath}=0$ for all $i \geq k$ and $R_{k-1} \neq 0$. Hence there exists $j \in \operatorname{supp}(S)$ such that $f\left(R_{k-1}\right)=S$.

CLAIM. $S_{\imath}=0$ for all $i \geq j+1$.

Suppose to the contrary that there exists $t \geq j+1$, such that $S_{t} \neq 0$. Then there exists $m \in \operatorname{supp}(R)$ such that $f\left(R_{m}\right)=S_{t}$. Clearly, $m<k-1$ and since $f$ is order-preserving we have $t<j$. But $t \geq j+1$ implies $j+1<j$ a contradiction. Therefore, $S_{z}=0$ for all $i \geq j+1$, i.e., $S$ is right limited. The converse is obvious. The proposition in the case of $R$ is left limited, can be similarly proved.

ACKNOWLEDGMENT. The authors would like to thank Professor H. Nakar from Aleppo University for his helpful suggestions.

\section{REFERENCES}

[1] REFAI, M and OBIEDAT, M., On graduations of $k\left[x_{1}, \ldots, x_{m}\right]$, J. Inst. Math. and Comp. Sci. (Math Series) 6(3) (1993), 241-252.

[2] REFAI, M., On Noetherian modules graded by G-sets, Acta Math. Hungarica 69(3) (1995), 211219.

[3] REFAI, M., Various types of strongly G-graded rings, Alexandria Engr. J. 32(2) (1993), 91-95.

[4] DADE, E.C., Group-graded rings and modules, Math. Z. 174 (1980), 241-262.

[5] NĂSTĂSESCU, C. and VAN OYSTAEYEN, F., Graded ring theory, Math. Library (28), NorthHolland, Amsterdam (1982). 


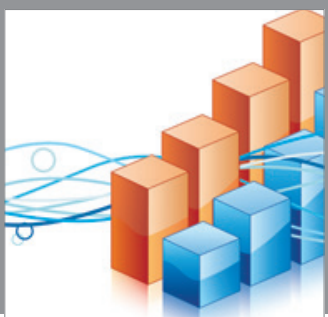

Advances in

Operations Research

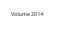

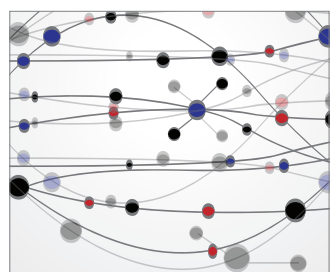

\section{The Scientific} World Journal
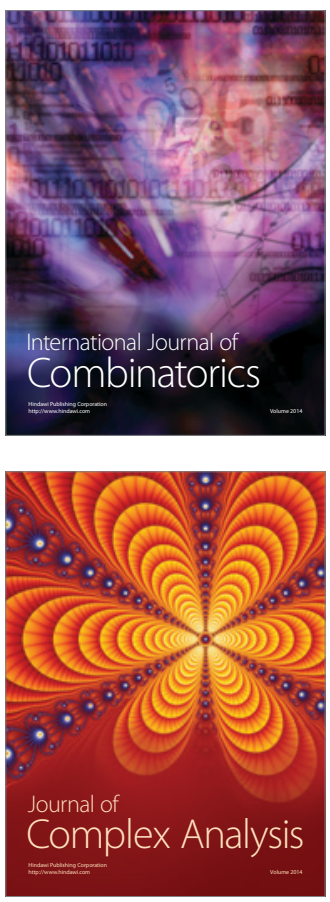

International Journal of

Mathematics and

Mathematical

Sciences
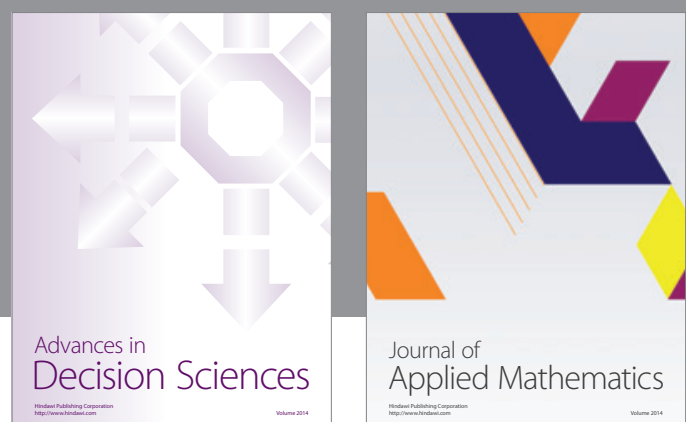

Journal of

Applied Mathematics
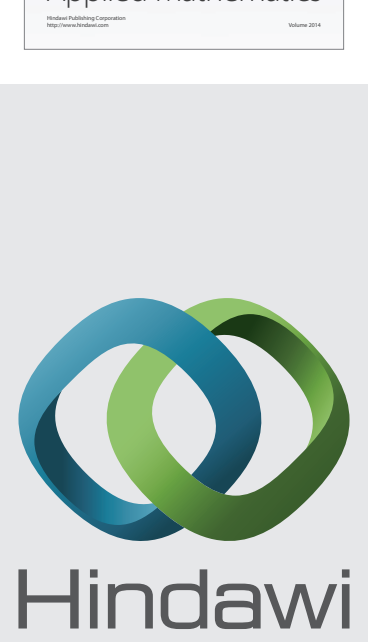

Submit your manuscripts at http://www.hindawi.com
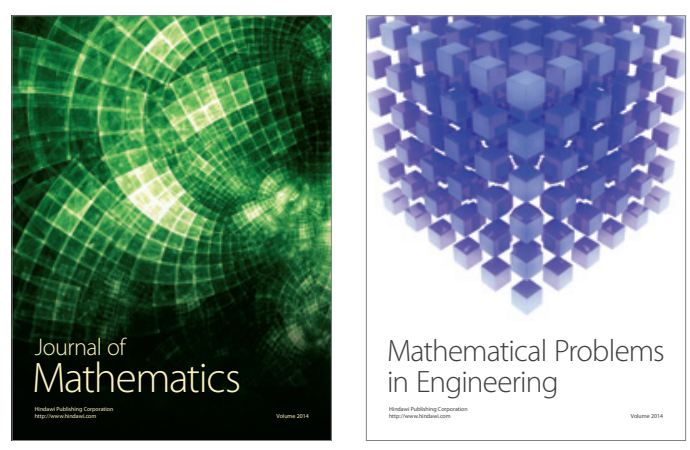

Mathematical Problems in Engineering
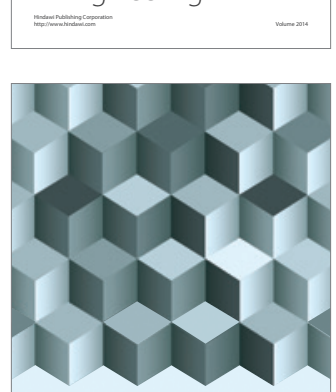

Journal of

Function Spaces
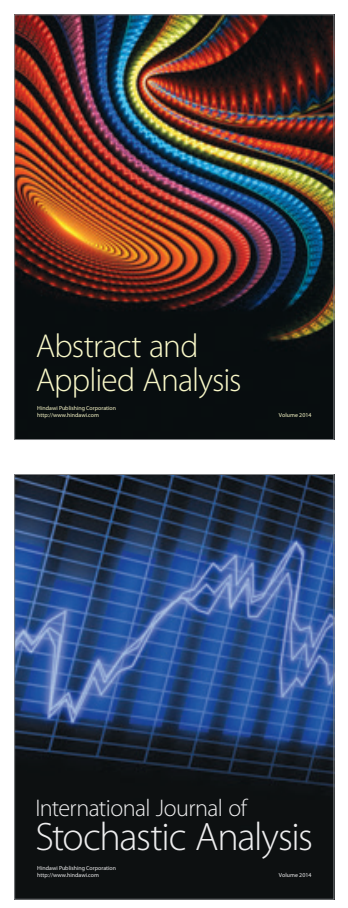

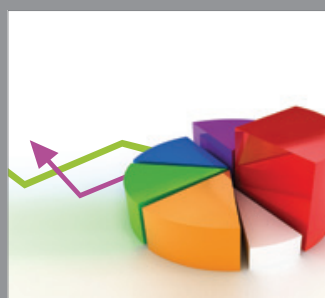

ournal of

Probability and Statistics

Promensencen
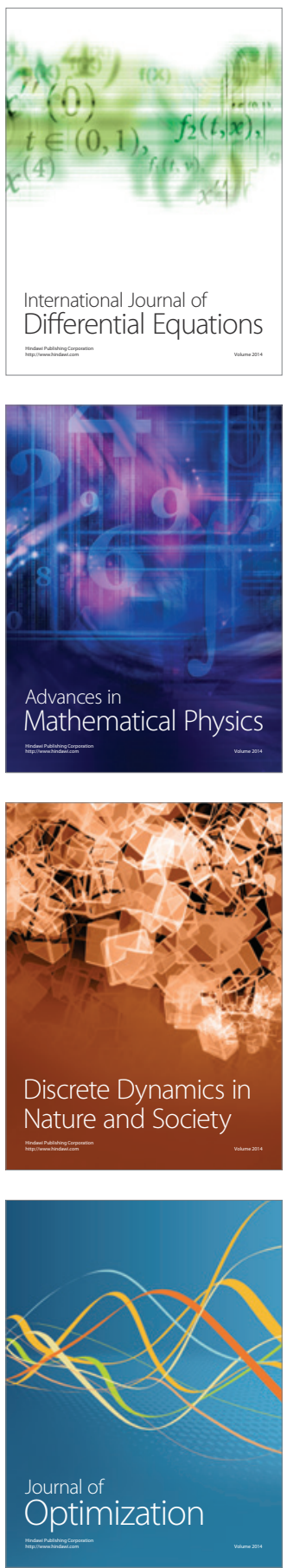